\title{
Metagenomics with guts
}

\section{Magdalena Zarowiecki}

This month's Genome Watch describes two recent studies that used metagenomic data to characterize the role of the human gut microbiota in disease.

Interactions between the gut microbiota and the immune system have an important role in many diseases both within and outside the gut $^{1}$. Two large collaborative efforts aimed at characterizing the human microbiome have concluded this summer (2012), after producing a string of high-profile papers ${ }^{1}$. The Metagenomics of the Human Intestinal Tract (MetaHIT) project sequenced the microbiota of faecal samples from both sick and healthy individuals and was discussed in a previous Genome Watch ${ }^{2}$. The Human Microbiome Project (HMP) focused mainly on characterizing the diversity of the microbiota in healthy humans and provides a baseline for the composition of the 'normal' gut microbiota $^{3}$. Although these studies have revealed many interesting correlations between the gut microbiota and disease, additional studies are required to identify the causal links underpinning these correlations.

A step towards understanding such correlations was taken by a systems-level network analysis of metagenomic data from the HMP and MetaHIT projects ${ }^{4}$. After constructing community-wide metabolic networks from the metagenomic sequence reads, the authors compared enzyme abundance between healthy individuals and individuals with obesity or inflammatory bowel disease (IBD), and identified those enzymes that were either enriched or depleted in individuals with these diseases. The disease-associated enzymes were found to be uncommon in the genomes of prevalent gut commensals, indicating that altered gut metabolism in patients with obesity and IBD is associated with only a small subset of rare microbial species. The topological position of the disease-associated enzymes in a given metabolic network was determined, revealing that these enzymes occupy either the start or end point of metabolic pathways more often than expected and are therefore likely to alter the range of metabolites in the gut because they either directly use or directly produce metabolites in this environment.

Bacteria have been the focus of most microbiome studies, but it has also been suggested that fungi, which are less abundant than bacteria in the intestine, can influence gut health. Using 454 and Illumina technology, Iliev et al..$^{5}$ characterized the fungal population of the mouse gut by sequencing a fragment of the internal transcribed spacer regions (ITS1 and ITS2) of fungal rRNA genes and showed that the mouse gut contains $>100$ species of fungi from $>50$ genera. The most common species was the opportunistic pathogen Candida tropicalis, and in a series of elegant experiments the authors demonstrated that this species interacts with the host immune system through the innate immune receptor DECTIN1 (also known as CLEC7A). They conclusively showed that in the absence of this receptor, the immune system cannot control C. tropicalis, which promotes intestinal inflammation. This suggests that fungi, in addition to bacteria, are important determinants of intestinal health. The authors also detected a strong association between severe ulcerative colitis and a single-nucleotide polymorphism in the human DECTIN1 gene, suggesting that fungi also have a role in IBD in humans.

In-depth analyses of metagenomic sequencing data, combined with experimental data, are now starting to provide deeper insights into the complex ecosystem of the gut. Such insights are important, as current methods of manipulating the gut microbiota

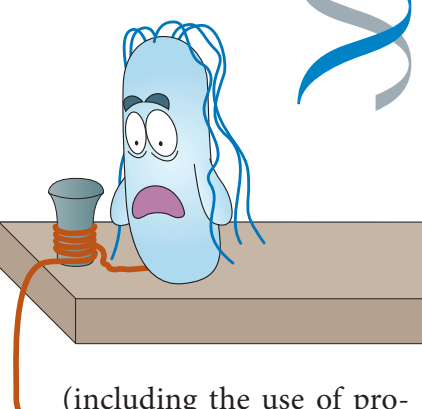

(including the use of probiotics, antibiotic treat-

ment or self-infestation with parasitic helminth worms) usually have broad-spectrum effects, the outcome of which is difficult to predict ${ }^{6}$. The hope is that advances in metagenomic analysis will ultimately lead to more specific manipulation of the gut microbiota in order to prevent and potentially cure many diseases ${ }^{1}$. Now, that certainly would give a good gut feeling.

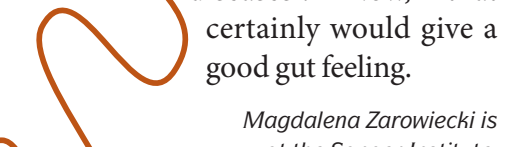
at the Sanger Institute, Wellcome Trust Genome Campus, Hinxton, Cambridge CB10 1SA, UK. e-mail:microbes@sanger.ac.uk doi:10.1038/nrmicro2879 Published online 10 September 2012

1. Cho, I. \& Blaser, M. J. The human microbiome: at the interface of health and disease. Nature Rev. Genet. 13, 260-270 (2012).

2. Walker, A. A glut from the gut: metagenomics takes a giant step foward. Nature Rev. Microbiol. 8, 315 (2010).

3. The Human Microbiome Project Consortium. Structure, function and diversity of the healthy human microbiome. Nature 486, 207-214 (2012).

4. Greenblum, S. et al. Metagenomic systems biology of the human gut microbiome reveals topological shifts associated with obesity and inflammatory bowel disease. Proc. Natl Acad. Sci. USA 109, 594-599 (2012).

5. Iliev, I. D. et al. Interactions between commensal fungi and the C-type lectin receptor Dectin-1 influence colitis. Science 336, 1314-1317 (2012).

6. Wolff, M. J. et al. Helminthic therapy: improving mucosal barrier function. Trends Parasitol. 28, 187-194 (2012).

Competing interests statement

The author declares no competing financial interests. 